\title{
TOWARDS AUTOMATED TOTAL PROCESS IN ROAD CONSTRUCTION
}

\author{
Rauno Heikkilä, Mika Jaakkola \\ Oulu University, Research of Construction Technology \\ P.O.Box 4400 (Kasarmintie 4), FIN-90014 Oulu, Finland \\ rauno.heikkila@oulu.fi,mika.jaakkola@oulu.fi
}

\begin{abstract}
Information technology can be generally understood as techniques and methods needed for creating, processing, refining, transferring and utilizing information through different working processes of construction. For managing information process different measurement, software, modeling, information communication as well as numeric control technologies are needed on the operational level. A visionary model of automated total process for future road construction is introduced. The main parts of the model have been tested by several different R\&D projects carried out in Finland 2001-2004.
\end{abstract}

Keywords: Construction, Total process, Information technology, automation.

\section{Introduction}

Full-scale integration of information technology into the civil construction process will in the future enable increasingly important innovations in the implementation of the individual phases of construction. The work process will digitized, the information will be organized and managed using 3-D geometric data, conventional work processes will be broken down into subprocesses, re-organized and mechanized in new ways, while the level of automation will increase to combine previously separate and unconnected operations to achieve smoother and more efficient cooperation (Fig. 1). At the same time, the work process is a generic model development process in the course of which the original models created out of input data measurements are upgraded, expanded and exploited throughout the operational process.

In the future, full-scale integration of information technology into the civil construction process enables increasingly important innovations in the implementation of the individual phases of construction. The work process will digitized, the information will be organized and managed using 3-D geometric data, conventional work processes will be broken down into subprocesses, re-organized and mechanized in new ways, while the level of automation will increase to combine previously separate and unconnected operations to achieve smoother and more efficient cooperation (Fig. 1). At the same time, the work process is a generic model development process in the course of which the original models created out of input data measurements are upgraded, expanded and exploited throughout the operational process (Heikkilä \& Jaakkola 2003, 2004).

\section{Suggesting a model for the total process of Road Construction}

The ICT process starts with input data measurements carried out on the site. In civil and house construction projects, the key input data includes variations in terrain and elevation and soil features. Over the past few years, laser scanning from an aircraft, helicopter or on the ground has developed greatly and become increasingly popular. A laser-scanned cloud of 3D points adjusted to the relevant coordinate system can be imported into a semi-automatic analysis application that can be used to model not only the contours of the terrain but also the tree stands, road alignments and buildings [Fig. 2]. However, 3D modeling of soil features and their conversion into a digitized format is technically far more complicated than terrain modeling. Theoretically, it should be fully possible. 


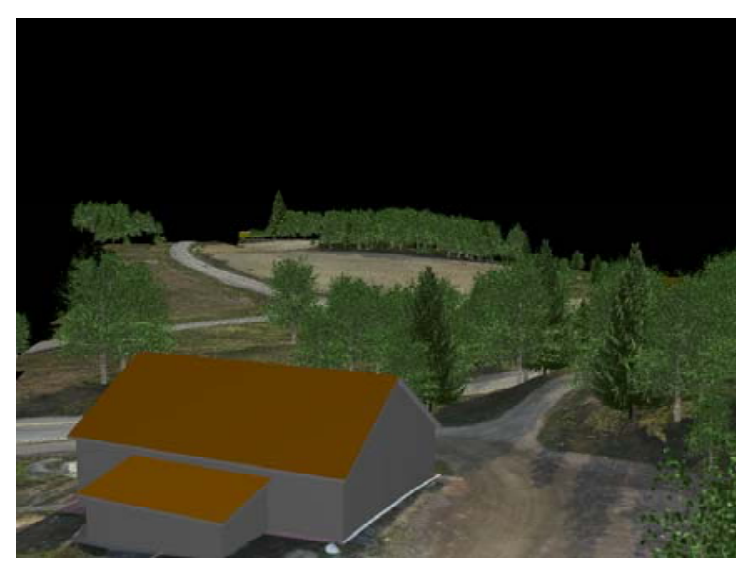

Fig. 2. Final result of input data measurements - an accurate $3 D$ model of the terrain.

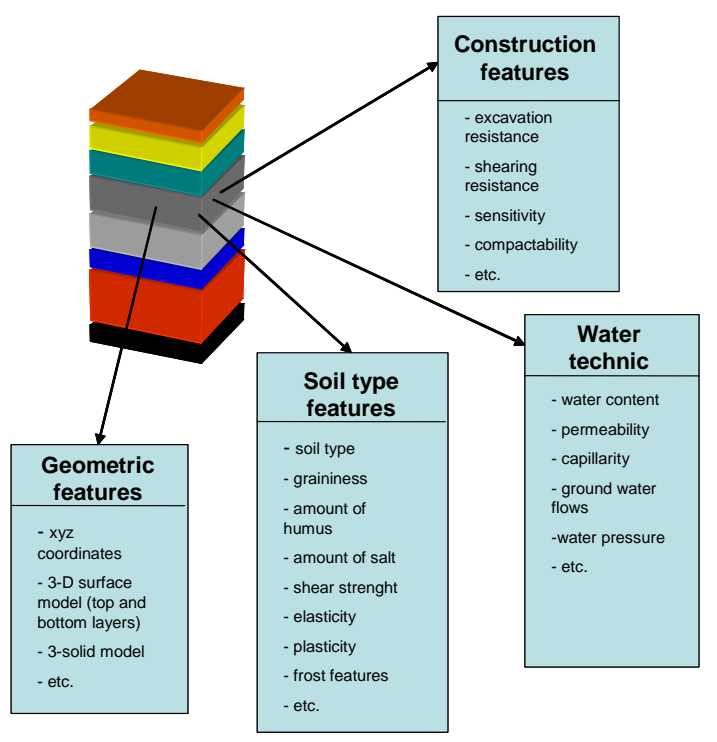

Fig. 3. Vision for the data content of a future n-dimensional terrain model.

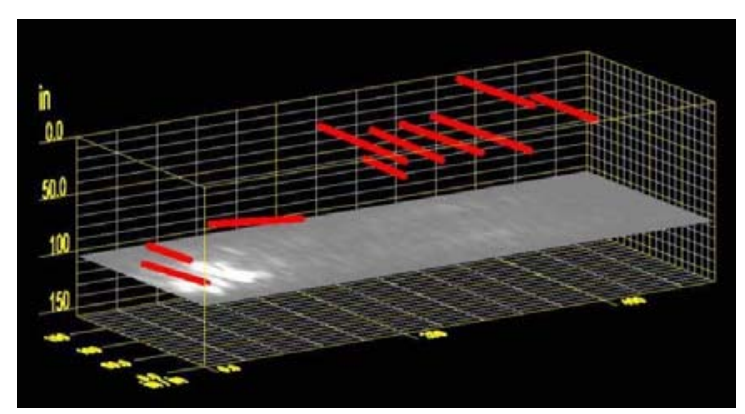

Fig. 4. Simplified example of a 3D model of existing structures.

In product design, a model and instructions for the implementation of the product are created out of the input date. Typically, the products are large 3-dimensional objects [Fig. 3]. If the input data is 3-dimensional, design can be carried out 3-dimensionally using CAD tools. Additionally, the 3D geometric model can be used to produce images for 2D drawings. With improved efficiency in design, it is also essential to be able to make use of the design model for construction purposes. If the geometric model is accurate and readily positioned in the site coordinate system, it can be used directly for controlling measurements and construction machines. This imposes additional requirements on the quality and accuracy of design. Similarly, an online connection between the CAD application and $3 \mathrm{D}$ measuring technique is feasible [Heikkilä \& Jaakkola 2002].

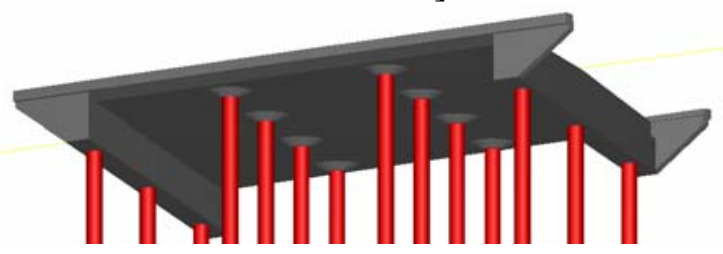

Fig. 5. 3-D product model of a bridge.

In the future, machines used in civil construction will be controlled by automated systems. As is known, the most advanced systems presently available permit partial automatic control of the road grader blade based on 3D positioning and 3D models. However, the functional performance of the systems varies, usually because they only make use of geometric data that is not managed completely. Full control of the 3D geometric data and, in particular, inclusion of other property data in the control system are still things of the future. Previous research and experiments indicate that the control of construction machines and blades requires active coordination by the operator. In this system, the blade position is adjusted automatically with reference to the control model, permitting the operator to select among various options to optimize the process according to the situation at hand. Evidently, pre-calculated paths of travel can seldom be followed. Typically, 3D point, 
curve and triangulated surface model models provide sufficient geometric control data for finishing surfaces. However, their data content is not enough for a control model for work operations such as the reinforcement of the road bed or stabilization of structural layers, where the objective is to modify the properties of the materials. For example, in pile driving, the pile may be broken when the soil type and its penetration resistance change as the pile pierces new layers. If the $3 \mathrm{D}$ geometric model provided information about penetration resistance, it would be possible to control the driving force when penetration resistance reaches its maximum while ensuring that the tensile stress inside the pile does not exceeded its capacity.

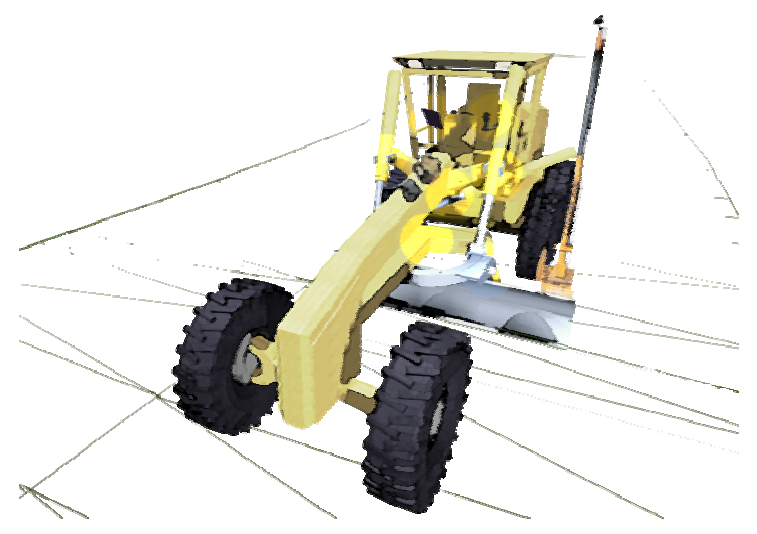

Fig. 6. A 3D control concept for a road grader.

Technology that improves the efficiency and standard of quality control is represented by ground-based 3D laser scanners. As a result of the 3D re-measurement of the bridge we obtain a cloud of $3 \mathrm{D}$ points that can be compared to the 3D design model and used for computing deviations in dimensions, form and position and evaluating them with reference to the tolerances determined according to functional and financial criteria.

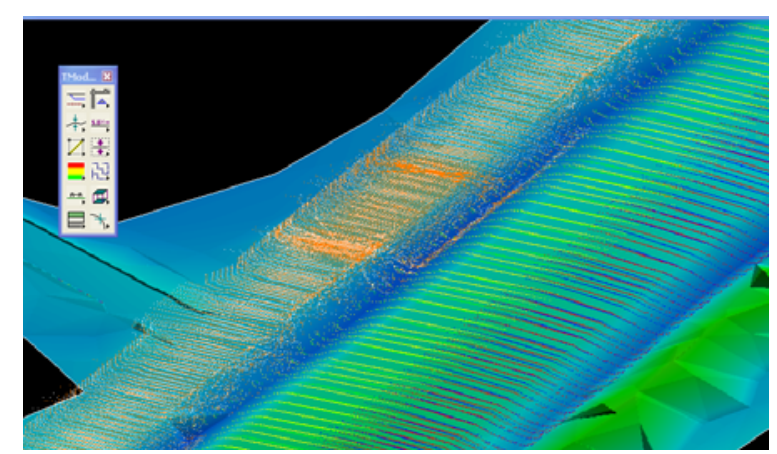

Fig. 7. Output from the measurement of road surface carried out with a new mobile laser scanning system developed in Finland.

\section{Conclusion}

Information as well as automation technology have a comprehensive impact on the overall construction process. New technologies can be applied and utilised throughout the road management processes in the acquisition of source information, product design, site management, quality inspection as well as upkeep and maintenance. The automated process of the future will be digital, three-dimensional, model-based, mechanised and automated. It will also be a process of model refinement where the model created at the beginning will be refined and utilised throughout the process. In this renovation we should try to understand and manage the current of innovations and changes bu modeling the total entirety.

\section{References}

Craig, D. \& Rajat, R. [2004]. Developing Customer-focused Culture in the Speculative House-building Industry. Total Quality Management, Vol.15, No. 1, January, pp. 73-87.

Haupt, T. \& Whiteman, D. [2004]. Inhibiting factors of implementing total quality management on construction sites. The TQM Magazine, Vol. 16, Number 3, pp. 166-173. 
Heikkilä, R. \& Jaakkola, M. (2002) The Efficiency of a 3-D Blade Control System in the Construction of Structure Layers by Road Grader - Automated Design-Build of Road Construction in Finland. ISARC'2002, 19th International Symposium on Automation and Robotics in Construction, 23-25 September 2002. Washington, DC, the United States of America, pp. 475-480.

Heikkilä, R. \& Jaakkola, M. [2003] Intelligent Road Construction Site Development of Automation into total working Process of Finnish Road Construction. ISARC'2003, 20th International Symposium on Automation and Robotics in Construction, 21-24 September 2003. Eindhoven, the Netherlands, pp. 265-269.

Heikkilä, R. \& Jaakkola, M. [2004] Towards Model based Automation - Different types of 3-D Machine Control Models for the Automatic Control of Road Construction Machinery. ISARC'2004, 21st International Symposium on Automation and Robotics in Construction, 21-25 September 2004. Jeju, Korea, pp. 53-58.

Kanji, G. and Wong, A., [1998]. Quality culture in the construction industry, Total
Quality Management, Vol. 9, Number 4-5 pp. 133-140.

Savolainen, T. \& Heikkilä, R. [2001] Towards Partnership Quality in Construction: the Case of a Finnish Regional Industry Cluster. Paisley, The $6^{\text {th }}$ ICIT, Sixth International Conference on ISO 9000 \& TQM in Ayr, Scotland, 17-19 April 2001, pp. 500-504.

Savolainen, T. \& Heikkilä R. [2002] Added Value for the Customer through an Innovative Total Delivery Process - a Proactive Network Approach to Construction of the Future. In: Co-operation \& Competition, the Organization of the Future. Proceedings of the 3rd International Conference on Co-operation \& Competition, C\&C, 19-21.9.2002, Växjö, Växjö University, Sweden. Mosad Zineldin [ed]. Lund, Sweden: Studentlitteratur, 2002:232237. - ISBN 91-7636-350-3.

Savolainen, T. \& Heikkilä, R. [2003] Hearing the Customer's Voice in Construction Process - Participation of Inhabitants in early Town Planning. Växjö, The $4^{\text {th }}$ International Conference on Cooperation and Competition [C \& C], Sweden, 11-13 September 2003, 10 p. 


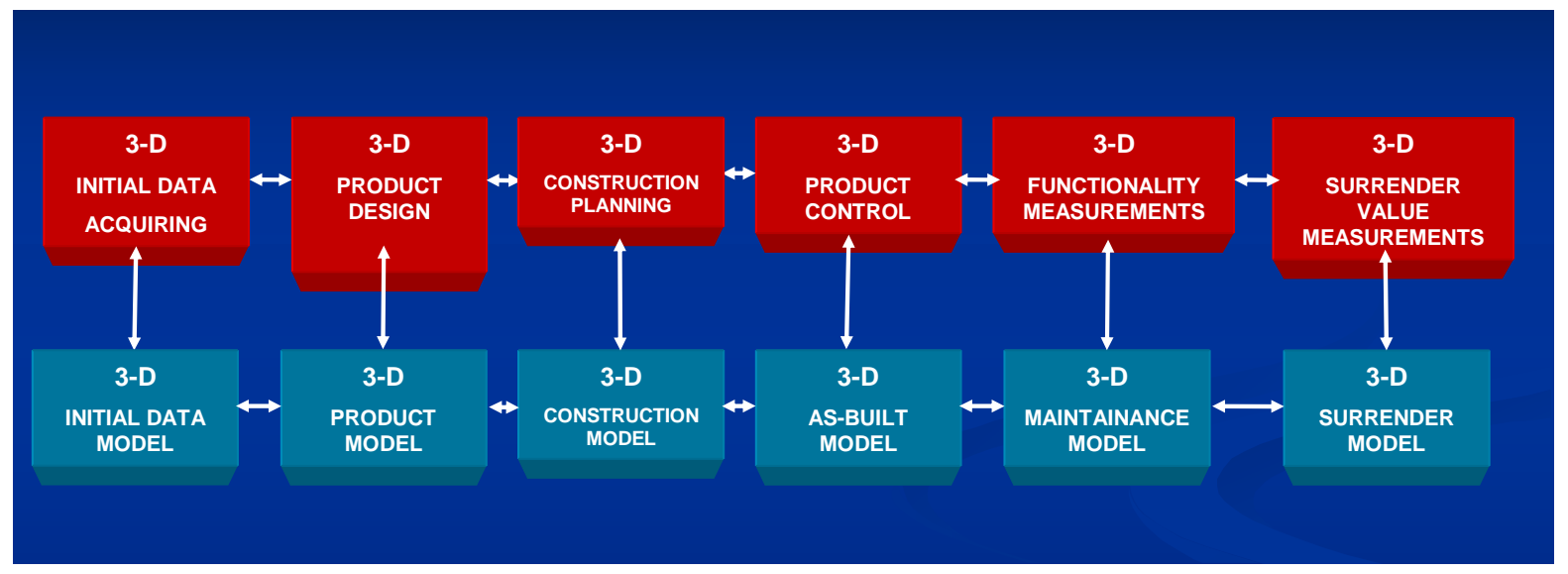

Fig. 1. Model of an Integrated IT Process in Construction.

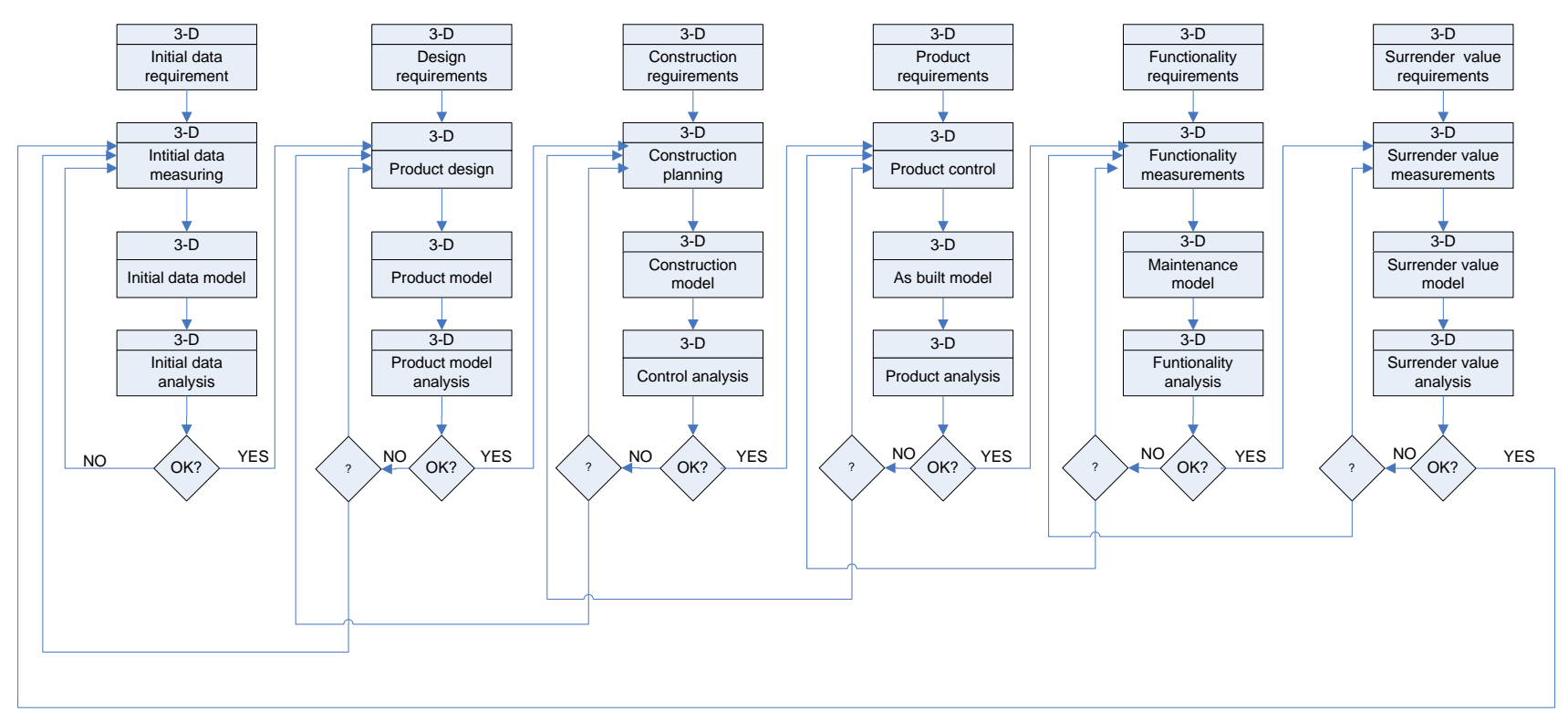

Fig. 2. An idea of the Total Quality Management principle in IT Process of Construction. 